\title{
Correspondence
}

Editor: Ian Pullen

Contents: First episode schizophrenia: predicting outcome/Gender-identity clinic patients/Musical and verbal hallucinations/Failure to convulse with ECT/Anorexia nervosa in the elderly/Chronic fatigue syndrome/Family intervention/Uraemia and mania/ The social networks of long-term patients/Incestuous abuse in psychiatric patients/Tolbutamide and fatal water intoxication/Tardive oculogyric crisis and obsessional thoughts/Drug-induced koro in a non-Chinese man.

\section{First episode schizophrenia: predicting outcome}

SIR: Prediction of outcome following first schizophrenic episode is an area of great importance since clinical practice is likely to be influenced by positive findings, especially if it is suggested that interventions such as drug-treatment can disadvantage occupational functioning in new patients suffering from schizophrenia with a pre-treatment duration of illness of less than a year. This finding has face validity but unfortunately the paper by Johnstone et al (Journal, August 1990, 157, 182-189) indicates the strength of this and all other associations only by providing a $P$ value. I find this a great pity, and surprising, in a paper which addresses associations and has performed a logistic regression analysis (which yields measures of association). All a $P$ value shows is the probability that an association could have been found by chance; it does not offer information about the strength of the association. If numbers are large, relatively low associations will be significant. The relevant statistical indicators are the correlations, and their confidence intervals between reported predictors and outcome, informing us of the amount of variation in outcome that can be explained by these predictors. I hope that these figures will be forthcoming, strengthening the importance of this study.

I was also unclear about the reason for the classification of all the unemployed as poor outcome, especially since the unemployed category contributed $88 \%$ of patients in the poor-outcome group, without any clarification as to whether these patients had always been unemployed, had become unemployed as a consequence of the illness before admission, or became unemployed after admission. An earlier paper reporting on the same patients (Johnstone et al, 1986) shows that 67 of the 253 patients $(26 \%)$ were unemployed on admission. Assuming this group remained unemployed, they would either form $67 \%$ of good outcome patients (being unchanged) or $50 \%$ of the poor outcome group (being unemployed). Either decision is debatable and requires a justification. Possibly a refinement of outcome criteria is indicated, since such a homogeneous classification of unemployed patients may explain some of the significant correlations, and may have led to important but potentially misleading conclusions. I am looking forward to a clarification on this issue.

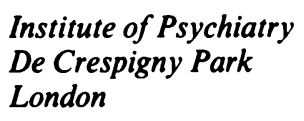

\section{Reference}

Johnstone, E. C., Crow, T. J., Johnson, A. L., et al (1986) The Northwick Park study of first episodes of schizophrenia: I. presentation of the illness and problems relating to admission. British Journal of Psychiatry, 148, 115-120.

AUTHORS' REPLY: We are grateful to Dr Muijen for his interest in our work and are sorry that he has found some aspects less than clear.

We are aware of the difficulties of statistical assessment in data like this, where a large number of variables are being assessed. We took this into account in our methods of calculation and mention the need for caution in the interpretation of data of this kind in the text. There are different views about the best means of dealing with this kind of data set, and it may help Dr Muijen to know that the analysis in the paper is a compromise between our own initial ideas and those of two referees whose views were to some extent conflicting. 